\title{
HOMOGENEOUS POLYNOMIALS AND INVARIANT SUBSPACES IN THE POLYDISC. II
}

\author{
TAKAHIKO NAKAZI AND KATSUTOSHI TAKAHASHI
}

(Communicated by Paul S. Muhly)

ABstract. We determine the invariant subspaces $M$ of $L^{2}\left(T^{2}\right)$ for which there is a subspace $S$ of $M$ and a positive integer $r$ such that

$$
M=\sum_{n=0}^{\infty} \bigoplus\left[\sum_{j=0}^{n} z^{j} w^{r(n-j)} S\right],
$$

where, for a subspace $A$ of $L^{2}\left(T^{2}\right),[A]$ denotes the closure of $A$.

\section{INTRODUCTION}

Let $L^{2}=L^{2}\left(T^{2}\right)$ be the Lebesgue space on the torus $T^{2}$, the product of two unit circles. A closed subspace $M$ of $L^{2}$ is said to be invariant if $z M \subseteq M$ and $w M \subseteq M$, where $z$ and $w$ are the coordinate functions on $T^{2}$. In a previous paper [3], the first author considered homogeneous decompositions for invariant subspaces (indeed, for invariant subspaces on a general torus) and determined invariant subspaces having such decompositions. In this paper we continue the study of [3].

Let $M$ be an invariant subspace of $L^{2}$ and let $r$ be a positive integer. For $n=0,1,2, \ldots$, put

$$
M_{n}=\left[\sum_{j=0}^{n} z^{j} w^{r(n-j)} M\right],
$$

where for a set $A$ of $L^{2},[A]$ denotes the closed linear span in $L^{2}$. Then $M_{n}$ is invariant and

$$
M=M_{0} \supseteq M_{1} \supseteq M_{2} \supseteq \cdots \supseteq M_{n} \supseteq \cdots .
$$

Thus, setting $S_{n}=M_{n} \ominus M_{n+1}$ for $n=0,1,2, \ldots$, we obtain the following

Received by the editors May 14, 1990 and, in revised form, September 4, 1990.

1980 Mathematics Subject Classification (1985 Revision). Primary 47A15, 46J15, 32A35.

The first author's research was partially supported by Grant-in-Aid for Scientific Research, Ministry of Education. 
decomposition:

$$
M=\left(\sum_{n=0}^{\infty} \bigoplus S_{n}\right) \oplus M_{\infty},
$$

where $M_{\infty}=\bigcap_{n=0}^{\infty} M_{n}$, which is an invariant subspace with $M_{\infty}=\left[z M_{\infty}+\right.$ $\left.w^{r} M_{\infty}\right]$. Clearly, for $n=0,1,2, \ldots$,

$$
z S_{n} \subseteq\left(\sum_{j=n+1}^{\infty} \bigoplus S_{j}\right) \oplus M_{\infty} \quad \text { and } \quad w^{r} S_{n} \subseteq\left(\sum_{j=n+1}^{\infty} \bigoplus S_{j}\right) \oplus M_{\infty} .
$$

Definition. Let $M$ be an invariant subspace of $L^{2}$ and $r$ a positive integer. If

$$
z S_{n} \subseteq S_{n+1} \text { and } w^{r} S_{n} \subseteq S_{n+1}
$$

for $n=0,1,2, \ldots$, then the decomposition $(*)$ is said to be $r$-homogeneous. If in addition $M_{\infty}=\{0\}$, then we call $M$ an $r$-homogeneous invariant subspace.

It is easy to see that $M=\left[z H^{2}+w^{r} H^{2}\right]$ is an $r$-homogeneous invariant subspace, where $H^{2}=H^{2}\left(T^{2}\right)$ is the space of all $f$ in $L^{2}$ whose Fourier coefficients $\hat{f}(j, k)$ are zero for every pair $(j, k)$ such that $j<0$, or $k<0$. We characterize $r$-homogeneous invariant subspaces. The case of $r=1$ was considered in [3], but the general case requires a new idea.

\section{Results}

Lemma 1. Let $M$ be an invariant subspace of $L^{2}$. If the decomposition $(*)$ is $r$-homogeneous, then the following relations hold.

(1) $w^{j} S_{0} \subseteq S_{0} \oplus S_{1}$ for $j=0,1,2, \ldots, r-1$.

(2) $S_{n}=\left[\sum_{j=0}^{n} z^{j} w^{r(n-j)} S_{0}\right]$ for $n=0,1,2, \ldots$.

Proof. Since the decomposition (*) is $r$-homogeneous, $w^{r} S_{0}$ is orthogonal to

$$
M_{2}=\left[z^{2} M+z w^{r} M+w^{2 r} M\right] .
$$

Hence, for $j=0,1,2, \ldots, r-1, w^{j} S_{0} \perp \bar{w}^{r-j} M_{2}$. Then, since $\bar{w}^{r-j} M_{2} \supseteq$ $M_{2}$, we have $w^{j} S_{0} \perp M_{2}$ and $w^{j} S_{0} \subseteq S_{0} \oplus S_{1}$. This shows (1). The assumption that $(*)$ is $r$-homogeneous also yields $\sum_{j=0}^{n} z^{j} w^{r(n-j)} S_{0} \subseteq S_{n}$ for $n=0,1,2, \ldots$. Since

$$
\sum_{j=0}^{n+1} z^{j} w^{r(n+1-j)} M=\sum_{j=0}^{n} z^{j} w^{r(n-j)}\left(z M+w^{r} M\right),
$$

it follows that

$$
\left[\sum_{j=0}^{n} z^{j} w^{r(n-j)} S_{0}\right] \oplus\left[\sum_{j=0}^{n+1} z^{j} w^{r(n+1-j)} M\right]=\left[\sum_{j=0}^{n} z^{j} w^{r(n-j)} M\right] .
$$

This shows (2). 
For a positive integer $r$, let $\mathscr{L}_{r}$ denote the subspace of all functions $f$ in $L^{2}$ whose Fourier coefficients $\hat{f}(j, k)$ are zero for every pair $(j, k)$ such that $r j+k \neq 0$. Thus the subspace $\mathscr{L}_{r}$ is the closure in $L^{2}$ of polynomials in $\bar{z} w^{r}$ and $z \bar{w}^{r}$. Let $S$ be a subspace of $\sum_{j=0}^{r-1} \oplus w^{j} \mathscr{L}_{r}$ and let $H_{S}$ denote the invariant subspace generated by $S$. The space $S$ is orthogonal to $\left[z S+w^{r} S\right]$. If

$$
w S \subseteq S \oplus\left[z S+w^{r} S\right]
$$

(note that this inclusion always holds for $r=1$ ), then $H_{S}$ has the decomposition

$$
H_{S}=\sum_{n=0}^{\infty} \bigoplus\left[\sum_{j=0}^{n} z^{j} w^{r(n-j)} S\right] \text {. }
$$

Thus $H_{S}$ is $r$-homogeneous and

$$
S_{0}:=H_{S} \ominus\left[z H_{S}+w^{r} H_{S}\right]=S .
$$

Theorem 1. If $M$ is an r-homogeneous invariant subspace of $L^{2}$ then $M=q H_{S}$, where $q$ is a unimodular function on $T^{2}$ and $S$ is a subspace of $\sum_{j=0}^{r-1} \bigoplus w^{j} \mathscr{L}_{r}$ such that

$$
w S \subseteq S \oplus\left[z S+w^{r} S\right] .
$$

Theorem 1 follows from Lemma 2 and Theorem 2 below.

Lemma 2. Let $M$ be an r-homogeneous invariant subspace and

$$
\widetilde{M}=\left[\bigcup_{k=0}^{\infty}\left(\bar{z} w^{r}\right)^{k} M\right] .
$$

Then $\widetilde{M}$ is an $r$-homogeneous invariant subspace. If

$$
M=\sum_{n=0}^{\infty} \bigoplus S_{n} \text { and } \widetilde{M}=\sum_{n=0}^{\infty} \bigoplus \widetilde{S}_{n}
$$

are the r-homogeneous decompositions of $M$ and $\widetilde{M}$, respectively, then

$$
\widetilde{S}_{n}=\left[\sum_{k=0}^{\infty}\left(\bar{z} w^{r}\right)^{k} S_{n}\right]
$$

for $n=0,1,2, \ldots$.

Proof. Obviously $\widetilde{M}$ is invariant. Let $R_{n}=\left[\bigcup_{k=0}^{\infty}\left(\bar{z} w^{r}\right)^{k} S_{n}\right]$ for $n=0,1$, $2, \ldots$ Since $M$ is $r$-homogeneous, if $m \neq n$, then $\left(\bar{z} w^{r}\right)^{k} S_{m} \perp\left(\bar{z} w^{r}\right)^{j} S_{n}$ for $k, j=0,1,2, \ldots$, so $R_{m} \perp R_{n}$. Hence we have $\widetilde{M}=\sum_{n=0}^{\infty} \oplus R_{n}$. Since $S_{n}=\left[\sum_{j=0}^{n} z^{j} w^{r(n-j)} S_{0}\right]$ by Lemma 1, we also have $R_{n}=\left[\sum_{j=0}^{n} z^{j} w^{r(n-j)} R_{0}\right]$. Thus $\widetilde{S}_{n}=R_{n}$ for $n=0,1,2, \ldots$. It is easy to see that $z R_{n} \subseteq R_{n+1}$ and $w^{r} R_{n} \subseteq R_{n+1}$ for $n=0,1,2, \ldots$. Therefore $\widetilde{M}$ is $r$-homogeneous. 
For a positive integer $r$, let $\mathscr{L}_{r}$ denote the closure in $L^{2}$ of the polynomials in $\bar{z} w^{r}$, a subspace of $\mathscr{L}_{r}$.

Theorem 2. Let $M$ be an r-homogeneous invariant subspace of $L^{2}$ such that $\bar{z} w^{r} M \subseteq M$.

(1) If $\bar{z} w^{r} M=M$, then $M=q H_{S}$ where $q$ is a unimodular function on $T^{2}$ and $S=\chi_{E}\left(\sum_{j=0}^{r-1} \oplus w^{j} \mathscr{L}_{r}\right)$ for some Borel set $E$ of $T^{2}$ whose characteristic function $\chi_{E}$ belongs to $\mathscr{L}_{r}$.

(2) If $\bar{z} w^{r} M \subsetneq M$ then $M=q H_{S}$, where $q$ is a unimodular function on $T^{2}$ and

$$
S=\left(\sum_{0 \leq j \leq i-1} \bigoplus w^{j} \mathscr{H}_{r}\right) \oplus\left(\sum_{1 \leq k \leq r-i} \bigoplus z \bar{w}^{k} \mathscr{H}_{r}\right)
$$

for some integer $i$ with $1 \leq i \leq r$.

If an invariant subspace $M$ satisfies $\bar{z} w^{r} M \subseteq M$, then for $n=1,2, \ldots$,

$$
\left[\sum_{j=0}^{n} z^{j} w^{r(n-j)} M\right]=z^{n} M
$$

and hence the decomposition $(*)$ for $M$ coincides with the Wold decomposition of $M$ with respect to the isometry of multiplication by $z$ on $M$, i.e.,

$$
S_{n}=z^{n}(M \ominus z M)
$$

for $n=0,1,2, \ldots$ If, moreover, the decomposition $(*)$ is $r$-homogeneous, then $w^{r}(M \ominus z M) \subseteq z(M \ominus z M)$. Conversely, we see that the condition $w^{r}(M \ominus z M) \subseteq z(M \ominus z M)$ for an invariant subspace $M$ implies that $\bar{z} w^{r} M \subseteq$ $M$ and $M$ has an $r$-homogeneous decomposition. Thus Theorem 2 should be compared with the results of [1,2], in which the condition $w(M \ominus z M) \subseteq$ $M \ominus z M$ for an invariant subspace $M$ was considered to characterize an invariant subspace of the form $q H^{2}$.

In the following we prove Theorem 2. Let $M$ be an $r$-homogeneous invariant subspace such that $\bar{z} w^{r} M \subseteq M$, and let $M=\sum_{n=0}^{\infty} \oplus S_{n}$ be the $r$-homogeneous decomposition of $M$. Then, as seen above, $S_{n}=z^{n} S_{0}$ for $n=1,2, \ldots$ and $\bar{z} w^{r} S_{0} \subseteq S_{0}$. Since $M$ is equal to the invariant subspace generated by $S_{0}$ by Lemma 1, in order to prove Theorem 2, it suffices to show that the subspace $\bar{q} S_{0}$, for some unimodular function $q$, is equal to one of the subspaces $S$ in Theorem 2 .

Let $S_{0}^{(1)}=\left\{f \in S_{0}: w^{r-1} f \in S_{0}\right\}$. (Note that if $r=1$, then $S_{0}^{(1)}=S_{0}$.)

Since Theorem 2 was proved in [3] in the case of $r=1$, let $r \geq 2$ in the following.

Lemma 3. (1) $S_{0}^{(1)} \neq\{0\}$.

(2) $\bar{z} w^{r} S_{0}^{(1)} \subseteq S_{0}^{(1)}$.

(3) $w^{j} S_{0}^{(1)} \subseteq S_{0}$ for $1 \leq j \leq r-1$.

(4) $w^{i} S_{0}^{(1)} \perp w^{j} S_{0}^{(1)}$ for $0 \leq i<j \leq r-1$. 
Proof. (1) Since $w^{r} S_{0} \subseteq S_{1}$, it follows that $w S_{0} \nsubseteq S_{0}$. Let $f$ be a function in $S_{0}$ such that $w f \notin S_{0}$. As remarked above, $S_{1}=z S_{0}$. It follows from (1) of Lemma 1 that

$$
w f=g+z h
$$

for some functions $g$ and $h$ in $S_{0}$ with $h \neq 0$, so

$$
w^{r-1} g+z w^{r-1} h=w^{r} f \in z S_{0} .
$$

Then, since $w^{r-1} g$ and $w^{r-1} h$ belong to $S_{0} \oplus z S_{0}$ by Lemma $1, z w^{r-1} h \in z S_{0}$ and so $w^{r-1} h \in S_{0}$. Hence $h$ is a nonzero element of $S_{0}^{(1)}$.

(2) follows from the inclusion $\bar{z} w^{r} S_{0} \subseteq S_{0}$.

(3) For $1 \leq j \leq r-1$, since $z w^{r-1-j} M \subseteq z M, w^{r-1} S_{0}^{(1)}$ and $z w^{r-1-j} M$ are orthogonal, hence $w^{j} S_{0}^{(1)} \perp z M$. This shows that $w^{j} S_{0}^{(1)} \subseteq S_{0}$.

(4) For $i<j$, since $w^{r} S_{0}^{(1)} \subseteq z S_{0}$ and $w^{r-j+i} S_{0}^{(1)} \subseteq S_{0}$ by (3),

$$
w^{r-j+i} S_{0}^{(1)} \perp w^{r} S_{0}^{(1)}
$$

and so $w^{i} S_{0}^{(1)} \perp w^{j} S_{0}^{(1)}$.

Lemma 4. (1) If $\bar{z} w^{r} S_{0}^{(1)}=S_{0}^{(1)}$, then $S_{0}^{(1)}=q \chi_{E} \mathscr{L}_{r}$ where $q$ is unimodular and $\chi_{E} \in \mathscr{L}_{r}$.

(2) If $\bar{z} w^{r} S_{0}^{(1)} \subsetneq S_{0}^{(1)}$, then $S_{0}^{(1)}=q \mathscr{H}_{r}$ where $q$ is unimodular.

Using Lemma 3 , the proof is completed by the same argument as in the proofs of [3, Theorem 3 and Proposition 5].

By Lemma 3, we have $S_{0} \supseteq \sum_{j=0}^{r-1} \bigoplus w^{j} S_{0}^{(1)}$.

Lemma 5. Let $S_{0}^{(2)}=S_{0} \ominus\left(\sum_{j=0}^{r-1} \oplus w^{j} S_{0}^{(1)}\right)$. If $f \in S_{0}^{(2)}$, then

$$
f=\sum_{j=1}^{r} z \bar{w}^{j} g_{j}
$$

where $g_{j} \in S_{0}^{(1)} \ominus \bar{z} w^{r} S_{0}^{(1)}$ for $1 \leq j \leq r$ and the functions $g_{j} \quad(1 \leq j \leq r)$ satisfy

$$
w^{k}\left(\sum_{j=k+1}^{r} z \bar{w}^{j} g_{j}\right) \in S_{0}^{(2)}
$$

for $0 \leq k \leq r-1$.

Proof. By Lemma 1, we have $w f=f_{1}+z g_{1}$ for some $f_{1}$ and $g_{1}$ in $S_{0}$. Then we see that $f_{1} \in S_{0}^{(2)}$ and $g_{1} \in S_{0}^{(1)} \ominus \bar{z} w^{r} S_{0}^{(1)}$. In fact, since $f \in S_{0}^{(2)}, w f$ is orthogonal to $w^{j} S_{0}^{(1)}$ for $1 \leq j \leq r$. Hence $f_{1} \perp w^{j} S_{0}^{(1)}$ for $1 \leq j \leq r-1$ and $g_{1} \perp \bar{z} w^{r} S_{0}^{(1)}$. Since $w^{r} f \in z S_{0}$ and $w^{r-1} S_{0}^{(1)} \subseteq S_{0}$, it follows that $w f \perp S_{0}^{(1)}$ and so $f_{1} \perp S_{0}^{(1)}$. Thus $f_{1} \in S_{0}^{(2)}$. The proof of (1) in Lemma 3 shows that $g_{1} \in S_{0}^{(1)}$, hence $g_{1} \in S_{0}^{(1)} \ominus \bar{z} w^{r} S_{0}^{(1)}$. 
Now, assume that for some $k \geq 1$,

$$
w^{k} f=f_{k}+\sum_{j=1}^{k} z w^{k-j} g_{j}
$$

where $g_{j} \in S_{0}^{(1)} \ominus \bar{z} w^{r} S_{0}^{(1)}$ for $1 \leq j \leq k$ and $f_{k} \in S_{0}^{(2)}$. Then, using the argument given above, we have $w f_{k}=f_{k+1}+z g_{k+1}$ for $f_{k+1} \in S_{0}^{(2)}$ and $g_{k+1} \in$ $S_{0}^{(1)} \ominus \bar{z} w^{r} S_{0}^{(1)}$. Consequently,

$$
w^{k+1} f=f_{k+1}+\sum_{j=1}^{k+1} z w^{k+1-j} g_{j} .
$$

Thus by induction, (**) holds for all $k \geq 1$. Since $w^{r} f \in z S_{0}$, it follows from $(* *)$ for $k=r$, that

$$
f=\sum_{j=1}^{r} z \bar{w}^{j} g_{j}
$$

For $0 \leq k \leq r-1$, comparing $(* *)$ with

$$
w^{k} f=w^{k}\left(\sum_{j=1}^{k} z \bar{w}^{j} g_{j}\right)+w^{k}\left(\sum_{j=k+1}^{r} z \bar{w}^{j} g_{j}\right),
$$

we have

$$
w^{k}\left(\sum_{j=k+1}^{r} z \bar{w}^{j} g_{j}\right)=f_{k} \in S_{0}^{(2)} .
$$

This completes the proof.

Proof of Theorem 2. (1) By Lemma 5, we have $\bar{z} w^{r} S_{0} \subseteq \sum_{j=0}^{r-1} \oplus w^{j} S_{0}^{(1)}$. Since $\bar{z} w^{r} S_{0}=S_{0}$ by hypothesis, it follows that

$$
S_{0}=\sum_{j=0}^{r-1} \bigoplus w^{j} S_{0}^{(1)} \quad \text { and } \quad \bar{z} w^{r} S_{0}^{(1)}=S_{0}^{(1)}
$$

Then by. Lemma $4, S_{0}^{(1)}=q \chi_{E} \mathscr{L}_{r}$ where $q$ is unimodular and $\chi_{E} \in \mathscr{L}_{r}$. Therefore we have

$$
S_{0}=q \chi_{E}\left(\sum_{j=0}^{r-1} \bigoplus w^{j} \mathscr{L}_{r}\right)
$$

and $M=q H_{S}$ where $S=\chi_{E}\left(\sum_{j=0}^{r-1} \oplus w^{j} \mathscr{L}_{r}\right)$.

(2) If $\bar{z} w^{r} S_{0}^{(1)}=S_{0}^{(1)}$, then by Lemma 5 we have

$$
S_{0}=\sum_{j=0}^{r-1} \bigoplus w^{j} S_{0}^{(1)}
$$


and hence $\bar{z} w^{r} S_{0}=S_{0}$. Thus under our assumption, $\bar{z} w^{r} S_{0}^{(1)} \subsetneq S_{0}^{(1)}$. It follows from (2) of Lemma 4 that $S_{0}^{(1)}=q \mathscr{H}_{r}$ for some unimodular function $q$, and that $S_{0}^{(1)} \ominus \bar{z} w^{r} S_{0}^{(1)}=[q]$. Therefore, if

$$
f \in S_{0}^{(2)}=S_{0} \ominus\left(\sum_{j=0}^{r-1} \bigoplus w^{j} S_{0}^{(1)}\right)
$$

then by Lemma 5 there are scalars $\alpha_{j}(1 \leq j \leq r)$ such that

$$
f=q\left(\sum_{j=1}^{r} \alpha_{j} z \bar{w}^{j}\right)
$$

and

$$
w^{k}\left(q \sum_{j=k+1}^{r} \alpha_{j} z \bar{w}^{j}\right) \in S_{0}^{(2)}
$$

for $0 \leq k \leq r-1$. Let $s$ be the largest number among numbers $j$ with $\alpha_{j} \neq 0$. Then the conditions $w^{k}\left(q \sum_{j=k+1}^{s} \alpha_{j} z \bar{w}^{j}\right) \in S_{0}^{(2)}$, for $0 \leq k \leq s-1$, imply $q z \bar{w}^{j} \in S_{0}^{(2)}$ for $1 \leq j \leq s$ and so $s \leq r-1$. Thus we have

$$
S_{0}^{(2)}=q\left[\left\{z \bar{w}^{j}: j=1,2, \ldots, s\right\}\right]
$$

for some $s \leq r-1$ or $S_{0}^{(2)}=\{0\}$. It follows that

$$
\bar{q} S_{0}=\left(\sum_{0 \leq j \leq r-1-s} \bigoplus w^{j} \mathscr{H}_{r}\right) \oplus\left(\sum_{1 \leq k \leq s} \bigoplus z \bar{w}^{k} \mathscr{H}_{r}\right)
$$

for some $s=0,1,2, \ldots, r-1$, and that $M=q H_{S}$ where $S=\bar{q} S_{0}$.

\section{REFERENCES}

1. V. Mandrekar, The validity of Beurling theorems in polydiscs, Proc. Amer. Math. Soc. 103 (1988), 145-148.

2. T. Nakazi, Certain invariant subspaces of $H^{2}$ and $L^{2}$ on a bidisc, Canad. J. Math. 40 (1988), 1272-1280.

3. __ Homogeneous polynomials and invariant subspaces in the polydisc, Arch. Math. (to appear).

Department of Mathematics, Faculty of Science, Hokkaido University, Sapporo 060, JAPAN 